\title{
Respiratory Sinus Arrhythmia in Diabetic Neuropathy
}

\author{
J.D. Mackay \\ Diabetic Department, King's College Hospital, London, UK
}

Summary. Heart rate variation was measured at different rates of regular deep breathing ( 2.5 to 13 breaths $/ \mathrm{min}$ ) in 11 normal subjects and 13 diabetic patients with neuropathy, defined clinically by the absence of both ankle jerks or the presence of autonomic symptoms. Subjects were divided into those above and below 30 years old. Normal subjects showed twofold changes in heart rate variation with maximum variation at mean breathing rates of 6.3 and 5.5 breaths $/ \mathrm{min}$. Heart rate variation was significantly smaller than normal in the diabetic patients with neuropathy, at all of the frequencies tested in the younger group and 7 of the 13 test frequencies in the older group; the maximum variation occurred at slower mean breathing rates of 4.5 breaths $/ \min (p<0.005)$ and 4.1 breaths/ $\min (p<0.01)$ respectively. Seven diabetic patients without neuropathy, with intact leg reflexes and $<30$ years old, had normal heart rate variation but the maximum responses occurred at a mean breathing rate of 5.4 breaths/min, significantly slower than normal $(p<0.02)$. Measuring the breathing rate at which heart rate variation is maximal may be a sensitive way of detecting autonomic (vagal) neuropathy.

Key words: Respiratory sinus arrhythmia, heart rate, diabetes, autonomic neuropathy, vagus nerve.
Recent interest in diabetic autonomic neuropathy has been stimulated by the development of tests, mostly of cardiovascular function, which can detect and quantify abnormal reflex responses [1].

Factors causing variation in heart rate are complex $[2,3]$, but there is a clear relationship between heart rate variation (HRV) and breathing, in particular regular breathing, well known as respiratory sinus arrhythmia. Loss of the normal HRV on deep breathing has been used as a simple and sensitive test for diabetic autonomic neuropathy $[4,5]$ : when abnormal it indicates the presence of vagal neuropathy [4]. The routine test is performed at a standardized rate of 6 breaths/min; this rate was chosen since, in addition to convenience, the amplitude of HRV depends on respiratory rate and in normal subjects is maximal at $5-6$ breaths/ $\min [6-8]$.

The aim of this study was to measure HRV at different rates of breathing in normal subjects and to compare the results obtained with those from diabetic patients with and without neuropathy.

\section{Methods}

As the amplitude of HRV on deep breathing declines with age [5], normal and diabetic subjects were divided by age into two sets, each with a narrow age range: those $<30$ years old (age range $20-29$ years) and those 30 years old and over (age range $30-43$ years). In the younger set there were three groups: six normal subjects (group 1), seven diabetic patients without neuropathy (group 2) and five diabetic patients with neuropathy (group 3 ). The mean durations of diabetes were similar: group 2, 14.0 years (range: 6-21 years); group 3, 16.6 years (range: 10-23 years). In the older set there were only two groups: five normal subjects (group 4) and eight diabetic patients with neuropathy (group 5). The duration of diabetes in group 5 was 24.0 years (range: 9-35 years).

Within each set groups were well matched for age. Statistical comparisons were made between the groups in each set of subjects but not between the two age sets. No normal subject had known cardiovascular, respiratory or neurological disease and none was diabetic.

The 13 diabetic patients with neuropathy had either peripheral neuropathy (12 patients: $4 / 5$ in group $3,8 / 8$ in group 5), autonomic neuropathy (seven patients: $5 / 5$ in group $3,2 / 8$ in group 5), or both (six patients). Diabetic patients without neuropathy had intact leg reflexes and no autonomic symptoms. Both forms of neuropathy were defined clinically: peripheral neuropathy by the absence of both ankle jerks (peripheral nerve conduction studies were not performed); and autonomic neuropathy by the presence of autonomic symptoms. One patient had postural hypotension (fall in systolic blood pressure on standing of $>30 \mathrm{mmHg}$ ), four had diarrhoea of characteristic pattern and four had gustatory sweating.

All diabetics were receiving insulin but no other medication.

\section{Clinical Procedure}

Subjects were tested in the morning or afternoon. The diabetic patients received their normal insulin and diet. As assessed by clinic visits 
Table 1. Analysis of the maximum recorded amplitudes of heart rate variation (HRV) for normal subjects and diabetic patients with neuropathy (all ages combined)

\begin{tabular}{lllll}
\hline & & \multicolumn{3}{c}{$\begin{array}{l}\text { Maximum recorded amplitude of heart } \\
\text { rate variation (beats/min) }\end{array}$} \\
\cline { 4 - 5 } $\begin{array}{l}\text { Group } \\
\text { (all ages) }\end{array}$ & $\begin{array}{l}\text { Age } \\
\text { (years) }\end{array}$ & $\begin{array}{l}\text { Peak } \\
\text { heart rate }\end{array}$ & $\begin{array}{l}\text { Trough } \\
\text { heart rate }\end{array}$ & $\begin{array}{l}\text { Amplitude of } \\
\text { HRV (peak- } \\
\text { trough heart } \\
\text { rate) }\end{array}$ \\
\hline $\begin{array}{l}\text { Normal subjects } \\
\text { (n=11) }\end{array}$ & $30.3 \pm 5.9$ & $83.8 \pm 7.5$ & $62.2 \pm 8.1$ & $21.6 \pm 5.6$ \\
$\begin{array}{l}\text { Diabetic } \\
\text { patients with } \\
\text { neuropathy } \\
\text { (n=13) }\end{array}$ & $32.9 \pm 5.1$ & $84.4 \pm 6.7$ & $73.3 \pm 6.0$ & $11.1 \pm 4.5$ \\
\hline $\begin{array}{l}\text { Statistical } \\
\text { comparison }\end{array}$ & NS & NS & $p<0.005$ & $p<0.001$ \\
\hline
\end{tabular}

All results expressed as mean \pm SD. Statistical comparisons werc made using Student's t-test; NS = not significant

Normal subject Diabetic patient with neuropathy
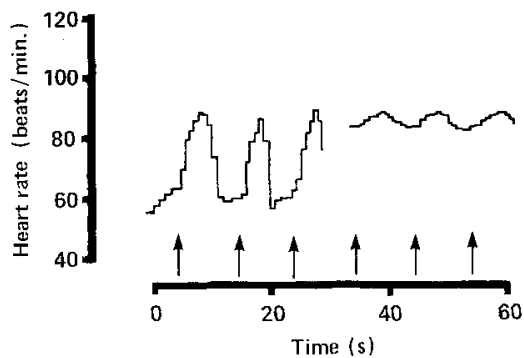

Fig. 1. Record of the cyclical changes in heart rate with regular deep breathing at 6 breaths $/ \mathrm{min}$ in a normal subject and a diabetic patient with neuropathy. Arrows indicate the onset of inspiration

their diabetes was stable; none became clinically hypoglycaemic during the test. All remained supine throughout; testing began after a 15 min rest. They were connected to an oscilloscope and a HewlettPackard 8020A heart rate monitor (Palo Alto, CA, USA) which recorded the beat-by-beat heart rate on paper. Heart rate recordings were made during episodes of regular deep breathing, the timing regulated by the experimenter using a clock. The subjects' depth of breathing was not recorded, but they were observed closely, encouraged to maintain deep breathing throughout and to inspire and expire smoothly. Tests were performed at 13 different rates of breathing from fast ( $15 \mathrm{breaths} / \mathrm{min}$ ) to slow ( 3 breaths $/ \mathrm{min}$ ). In 8 of the 13 diabetic patients with neuropathy testing was performed to even slower rate of breathing ( 2.5 breaths $/ \mathrm{min}$ ).

Eight cycles of breathing were recorded at each test frequency, thus taking $0.5-2.5 \mathrm{~min}$ to perform the test. There was a $2 \mathrm{~min}$ rest before each recording. Repeat measurements were obtained in 16 of the 31 subjects (five normal subjects, four diabetic patients without neuropathy, seven diabetic patients with neuropathy) by testing from slow to fast rates of breathing.

\section{Analysis}

Heart rate variation was assessed manually by measuring (from the beat-by-beat heart rate record) the difference between peak and trough heart rates over five deep breaths, taking the mean of ten consecutive readings (in beats $/ \mathrm{min}$, five from trough to peak, five from peak to trough) (Fig. 1) [5]. These measurements of the amplitude of
HRV were plotted against respiratory frequency (on a logarithmic scale) and frequency response curves drawn by eye. Two variables were derived: (1) the 'resonance amplitude', the maximum amplitude of HRV, in beats/min; (2) the 'resonance frequency', the frequency of respiration, in breaths/min, at which the maximum amplitude of HRV occurred. Mean frequency-response curves were obtained for the five groups: at each breathing rate tested the measurements of HRV made from the traces were pooled and the mean amplitude \pm SEM calculated.

\section{Statistical Analysis}

Statistical comparisons were made using Student's t-test and a 5\% level of significance accepted. However, in the younger set $(<30$ years old), to permit multiple comparisons between groups 1 to 3 , the test was modified by reducing the accepted level of significance to $2 \%$. This level of significance applied to comparisons of age and also the derived variables of resonance frequency and resonance amplitude.

Comparison of the mean amplitudes of HRV at each of 13 frequencies (3-15 breaths/ $\mathrm{min}$ ) between groups 1-3 in the younger set, and groups 4 and 5 in the older set, were made using a two-factor analysis of variance (factor $1=$ group, factor $2=$ frequencies) with repeated measures of the second factors [9]. From this the appropriate experimental error term was estimated and then the Tukey multiplecomparison procedure was used to obtain the least significant difference [10]. When two mean amplitudes for two groups of subjects measured at the same frequency differed by an amount greater than or equal to the least significant difference value the means were established as significantly different at the chosen significance level. The significance level was set at 5\%.

\section{Results}

Heart rate variation on deep breathing altered considerably between the different breathing rates; over the frequency range tested there was a two- to threefold change in amplitude. This pattern of response was seen in individual subjects and also, with particular clarity, when mean amplitudes at each test frequency were obtained for each of the five groups (Figs. 2 and 3). The smallest amplitudes were found at the very fast breathing rates. The amplitudes then increased evenly in a linear fashion with progressively slower breathing rates (using a logarithmic plot of respiratory frequency) until a maximum was reached; then they declined with the slowest breathing rates. Overall a frequency-response curve was obtained.

Young normal subjects (group 1) had a mean frequency response curve as described (Fig. 2) and a resonance frequency of $6.3 \pm 0.6$ breaths/min (mean \pm $1 \mathrm{SD})$. Age has effects on this response: the older group of normal subjects (group 4) overall had smaller amplitudes of HRV and the mean resonance frequency was at the slower rate of $5.5 \pm 0.3$ breaths/min (Fig. 3 ).

Diabetic patients without neuropathy (group 2) had similar amplitude responses to normal subjects (group 1) with no significant difference between their mean resonance amplitudes or between the mean amplitudes of HRV at the individual test frequencies (Fig. 2). Despite this, their frequency-response curves were shifted to the left with a mean resonance frequency of $5.4 \pm 0.3$ breaths $/ \mathrm{min}$, significantly slower than the normal subjects $(p<0.02)$. 


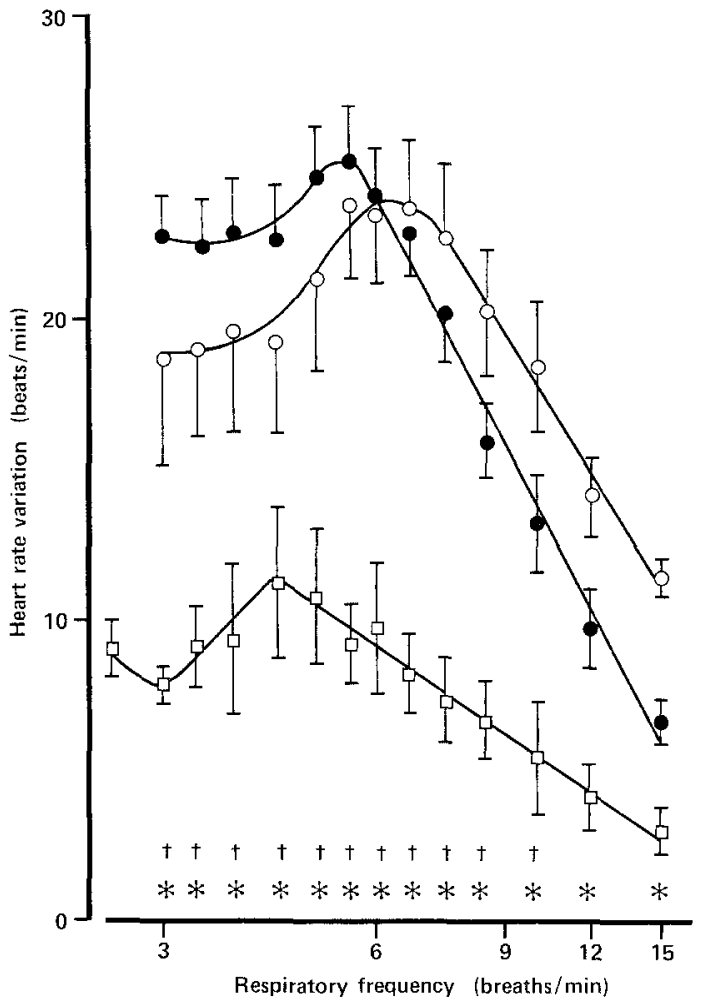

Fig. 2. Frequency-response curves in subjects $<30$ years old. The mean \pm SEM amplitudes of heart rate variation are plotted against $\log$ respiratory frequency for six normal subjects (group 1: $0-0$ ), seven diabetic patients without neuropathy (group 2: - - ) and five diabetic patients with neuropathy (group 3: $\square-\square$ ). Statistical comparison was by a two-factor analysis of variance. *denotes significant difference between groups 1 and 3 , and $\dagger$ between groups 2 and 3

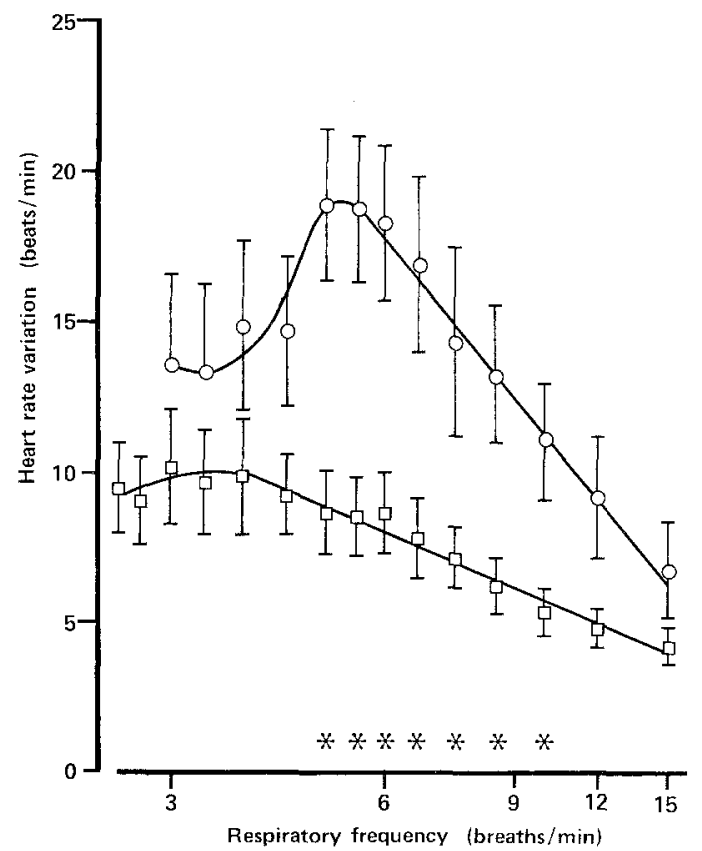

Fig. 3. Frequency-response curves in subjects $>30$ years of age. The mean \pm SEM amplitudes of heart rate variation are plotted against log respiratory frequency for five normal subjects (group 4: $0-0$ ) and eight diabetic patients with neuropathy (group 5: $\square-\square$ ). Statistical comparison was by a two-factor analysis of variance. * denotes significant difference in amplitude between groups 4 and 5
Diabetic patients with neuropathy, both young (group 3) and older (group 5), also showed an approximately twofold change in the amplitude of HRV within the frequency range studied, but overall the size of their responses was reduced (Figs. 2 and 3 ). The mean resonance amplitudes were significantly smaller than those of equivalent normal subjects (groups 1 versus $3, p<$ 0.005 ; groups 4 versus $5, p<0.02$ ), and also smaller than those of the diabetic patients without neuropathy (groups 2 versus $3, p<0.001$ ). Using a two-factor analysis of variance the young diabetic patients with neuropathy (group 3) had mean amplitudes of HRV significantly smaller than normal (group 1) at all 13 of the frequencies tested (Fig. 2), and the older subjects (groups 4 versus 5) at 7 of the 13 frequencies tested (Fig. 3). Discrimination between the heart rate responses of the diabetic patients with neuropathy and those of the normal subjects was best between the breathing rates of 5 and 7.5 breaths/min.

In addition to the reduction in the amplitude of HRV, diabetic patients with neuropathy showed a marked shift of the resonance frequencies to slower breathing rates (Figs. 2 and 3 ). The mean resonance frequency in those $<30$ years old (group 3) was $4.5 \pm 0.7$ breaths/min, significantly slower than normal subjects (group 1) $(p<0.005)$, and also slower than the diabetic patients without neuropathy (group $2 ; p<0.02$ ). The older diabetic patients with neuropathy (group 4) had a mean resonance frequency of $4.1 \pm 1.1 \mathrm{breaths} / \mathrm{min}$, significantly slower than the normal subjects (group 5; $p<0.01$.

Diabetic patients with neuropathy have smaller amplitudes of HRV because of less slowing of the heart rate during each respiratory cycle (Fig. 1). This is illustrated by analysis of the peak and trough heart rates at the maximum recorded amplitudes of HRV in all the 11 normal subjects and the 13 diabetic patients with neuropathy (Table 1). The mean peak heart rates are similar but the mean trough heart rates are significantly different; this entirely accounts for the difference in the amplitudes of HRV.

The heart rate response on testing from fast to slow rates of breathing (first series) was compared with the response on testing from slow to fast rates of breathing (second series) in the 16 subjects who had such studies performed. At each test frequency the 13 amplitudes from the first series were compared with the 13 amplitudes from the second series, using the paired $t$-test. No significant differences were detected at any of the test frequencies, suggesting that systematic errors were not developing during the procedure.

\section{Discussion}

Respiratory sinus arrhythmia, the variation of heart rate with respiration, may reflect a reflex adjustment of the heart to cyclic changes in systemic venous return in- 
duced by respiration [3]. Although the overall mechanisms are complex [2,3] efferent impulses are carried by the parasympathetic system [4]. Reduction of HRV on deep breathing may occur in diabetic patients $[4,5]$ and reflects underlying vagal neuropathy. In severe cases HRV on deep breathing is virtually abolished, and there may then be histological evidence of vagal denervation [11]. This study shows that the difference in the maximum recorded amplitudes of HRV between normal subjects and diabetic patients with neuropathy is due to the difference in the degree of slowing of the heart rate - a vagally mediated effect.

These experiments show that, in normal subjects resting supine, HRV on deep breathing is not constant but is frequency-dependent with an approximately twofold change in amplitude and a maximum response at about 6 breaths/min. Others have made similar observations [6-8] and have shown, by suitable monitoring of respiration, that the effect is a consequence of the frequency rather than the depth of respiration. In addition, it has been noted that heart rate changes were not significantly related to the depth of respiration provided tidal volumes were greater than $40 \%$ of the forced vital capacity [12].

There have been no previous studies of respiratory sinus arrhythmia in diabetic subjects at different breathing rates. Those diabetic patients without clinically detectable neuropathy had HRV amplitudes similar to those of normal subjects. Indeed, the mean amplitudes of $\mathrm{HRV}$ at 6 breaths/min, the standard rate of testing in previous studies $[4,5]$, were identical: However, the mean frequency-response curve was shifted to the left so that the mean resonance frequency was at a significantly slower breathing rate than normal.

The frequency-response curves of the diabetic patients with neuropathy showed two major features (Figs. 2 and 3). Firstly, although HRV increased twofold from fast to slower rates of breathing, the responses were significantly smaller than normal at most breathing rates. However, if single frequency testing is considered, measurement of HRV at 6 breaths/min discriminated as well between normal subjects and those with neuropathy as measurements at other breathing rates, and therefore remains useful for routine practice [5]. Secondly, the diabetic patients with neuropathy had maximum amplitudes of HRV at significantly slower breathing rates than the diabetic patients without neuropathy, and particularly so when compared with the normal subjects.

Thus this study identifies two abnormalities of HRV on deep breathing in selected diabetic patients, both of which are felt to reflect underlying autonomic (vagal) neuropathy. Firstly, as a new observation, there is a shift in the resonance frequency, that is the frequency at which the maximum amplitude of HRV occurs, to slower breathing rates. This suggests that the maximum rate of change of heart rate with time is slower than normal. The precise neurophysiological reason is uncertain. It may reflect delays in nerve conduction, or merely loss of coherence of neural impulses in a reflex system where synchrony is important. Such defects of temporal dispersion can occur when there are only minor changes in conduction velocity [13]. This change in the resonance frequency has been found even in young diabetic patients without clinically detectable neuropathy. It suggests that measurement of the resonance frequency may provide a sensitive way of detecting early autonomic (vagal) neuropathy. Secondly, there is reduction in the amplitude of HRV on deep breathing with more advanced neuropathy $[4,5,14,15]$. This may reflect a more severe pathology such as nerve fibre loss, which has been observed histologically in diabetic patients with severe autonomic neuropathy [11].

Acknowledgements. I am grateful to Dr. P.J. Watkins for his encouragement and support, and particularly to my wife, Dr. B.A. Mackay, for helpful scientific discussions. Statistical help was given by Mr. E. B. Farragher. Financial support was kindly provided by King's College Hospital Voluntary Research Trust Fund and by Sterling Winthrop Ltd.

\section{References}

1. Ewing DJ (1978) Cardiovascular reflexes and autonomic neuropathy. Clin Sci 55: $321-327$

2. Sayers B McA (1973) Analysis of heart rate variability. Ergonomics 16: 17-32

3. Melcher A (1976) Respiratory sinus arrhythmia in man. A study in heart rate regulating mechanisms. Acta Physiol Scand [Suppl] 435: $1-31$

4. Wheeler T, Watkins PJ (1973) Cardiac denervation in diabetes. $\mathrm{Br}$ Med J 4: 584-586

5. Mackay JD, Page M McB, Cambridge J, Watkins PJ (1980) Diabetic autonomic neuropathy. The diagnostic value of heart rate monitoring. Diabetologia 18: 471-478

6. Angelone A, Coulter NA (1964) Respiratory sinus arrhythmia: a frequency dependent phenomenon. J Appl Physiol 19:479-482

7. Womack BF (1971) The analysis of respiratory sinus arrhythmia using spectral analysis of digital filtering. IEEE Trans Biomed Eng 18: 399-409

8. Kelman GR, Wann KT (1971) Studies in sinus arrhythmia. J Physiol (Lond) 213: 59-60P

9. Wines BJ (1971) Statistical principles in experimental design, 2nd edn, McGraw-Hill, New York, pp 518-539

10. Wines BJ (1971) Statistical principles in experimental design, 2nd edn, McGraw-Hill, New York, pp 185-196

11. Duchen LW, Anjorin A, Watkins PJ, Mackay JD (1980) Pathology of autonomic neuropathy in diabetes mellitus. Ann Intern Med 92: 301-302

12. Bennet T, Farquhar ID, Hosking DJ, Hampton JR (1978) Assessment of methods for estimating autonomic nervous control of the heart in patients with diabetes mellitus. Diabetes 27:1167-1174

13. Gilliat RW, Willison RG (1962) Peripheral nerve conduction in diabetic neuropathy. J Neurol Neurosurg Psychiatry 25: 11-18

14. Sundkvist G, Almer L-O, Lilja B (1979) Respiratory influence on heart rate in diabetes mellitus. Br Med J 1: 924-925

15. Ewing DJ, Borsey DQ, Bellavere F, Clarke BF (1981) Cardiac autonomic neuropathy in diabetes: comparison of measures of $R-R$ interval variation. Diabetologia $21: 18-24$

Received: 5 May 1982

and in revised form: 15 November 1982

Dr. J.D. Mackay

Department of Medicine

University Hospital of South Manchester

Manchester M20 8LR, UK 